\title{
Detection of Ultra High-Energy Tau-Neutrinos with Fluorescence Detectors
}

\author{
C. A. Moura Jr. and M. M. Guzzo \\ Instituto de Física "Gleb Wataghin" - UNICAMP \\ 13083-970 Campinas SP, Brazil
}

Received on 6 January, 2007

\begin{abstract}
We calculate the possible number of Extensive Air Showers originated by tau neutrinos in Fluorescence Detectors like the ones of the Pierre Auger Observatory. We consider models of production of electron and muon neutrinos in extra galactic objects and Topological Defects, as well as the possibility of neutrino flavor change in the propagation of the neutrinos between the source and the Earth. The neutrino cross section was calculated by the extrapolation of the standard model parton distribution functions until energies of the order of $10^{21} \mathrm{eV}$. However, due to uncertainties in the extrapolation for energies higher than $10^{12} \mathrm{eV}$ the results are not robust. We conclude that, depending on the relation between flux and cross section, there is a strict range of energy for the tau neutrinos to generate double extensive air showers detectable in Fluorescence Detectors. The tau neutrino energy must be approximately $10^{18} \mathrm{eV}$ and the event rate can vary some orders of magnitude around one event per year, depending on the flux-cross section relation and detector characteristics.
\end{abstract}

Keywords: Neutrinos; Air showers; Pierre Auger Observatory

\section{INTRODUCTION}

It is believed that ultra-high energy (UHE) cosmic neutrinos may play an important role to explain the origin of cosmic rays with energies beyond the GZK limit of about $5 \times$ $10^{19} \mathrm{eV}[1,2]$, given that neutrinos hardly interact with cosmic microwave background or intergalactic magnetic fields, keeping their original energy and direction of propagation for very long distances. Even if they have masses or magnetic moments, or travel distances of the order of the visible universe, those characteristics do not change very much. Possible sources of these UHE neutrinos, like Active Galactic Nuclei and Gamma Ray Bursts, are typically located at thousands of $\operatorname{Mpc}[3,4]$.

Considering that neutrinos come from pions produced via the process $\gamma+p \rightarrow N+\pi$ [4], that there is an additional $v_{e}$ flux due to escaping neutrons and that about $10 \%$ of the neutrino flux is due to prompt decays, the proportionality of different neutrino flavors at the source results: $v_{e}: v_{\mu}: v_{\tau}=$ $0.6: 1.0:<0.01$ [5]. Nevertheless, observation of solar [6] and atmospheric [7] neutrinos present compelling evidence of neutrino flavor oscillations. Such oscillations have been independently confirmed by terrestrial experiments. KamLAND [8] observed $\overline{\mathrm{v}}_{e}$ disappearance confirming (assuming CPT invariance) what has been seen in solar neutrino detections and $\mathrm{K} 2 \mathrm{~K}[9,10]$ observed $v_{\mu} / \overline{\mathrm{v}}_{\mu}$ conversion compatible with what has been detected through atmospheric neutrino observations. When neutrino flavor oscillations are taken into consideration the flavor proportion will be modified to $v_{e}: v_{\mu}: v_{\tau} \sim 1: 1: 1$ [11]. Therefore one expects a considerable number of $v_{\tau}$ 's arriving at the Earth.

We investigate the possibility of detecting UHE $\operatorname{cosmic} v_{\tau}$ by means of a process in which a double Extensive Air Shower (EAS) is identified, the so-called Double-Bang (DB) Phenomenon. In that kind of event a $v_{\tau}$ interacts with a quark via charged current creating one cascade of hadronic particles and a tau lepton which subsequently decays producing a second cascade. DB Phenomenon was first proposed for detectors in which the neutrino energy should be around $1 \mathrm{PeV}$ [5]. It does not happen with neutrinos different from $v_{\tau}$. While the electron generated by an $v_{e}$ interacts immediately after being created, the muon generated by a $v_{\mu}$ travels a much longer distance than the size of the detector before interacting or even decaying. In fact, a muon with energy of the order of $1 \mathrm{EeV}$ will lose $36 \%$ of its initial energy after having crossed $36000 \mathrm{~g} / \mathrm{cm}^{2}$ in iron [12]. So we do not expect to have DB events from them.

In order to identify a DB Phenomenon in the atmosphere, an optical detector must be used to probe the longitudinal development of EAS's, recording the light emitted by the excited nitrogen molecules of the Earth's atmosphere when the EAS passes through it. A DB is triggered when two EAS's come from the same direction inside the field of view (f.o.v.) of the detector, i.e., in the physical space around the detector in which an event can be observed. We conclude that the features of optical detectors like the Fluorescence Detectors (FD's) used by the Pierre Auger Observatory [13] favor the observation of DB events with $v_{\tau}$ energies around $3 \mathrm{EeV}$. The estimated number of DB events to be observed in these FD's varies from a hundred in a year to few events in hundreds of years depending mainly on the assumed primary $v_{\tau}$ flux and cross section.

\section{THE ULTRA-HIGH ENERGY DOUBLE-BANG}

Studying the characteristics of FD's such as their efficiency and f.o.v., and the characteristics of the DB events generated by UHE $v_{\tau}$ 's, one can estimate the rate of this kind of event expected in that kind of detector.

Figure 1 shows a schematic view of an UHE DB with the detector position and the time integrated development of the two EAS's, the first one created by the UHE $v_{\tau}$ after having interacted with a nucleon in the atmosphere and the other one created by the decay of the tau generated in the first interaction of the $v_{\tau}$. The f.o.v. of the Pierre Auger Observatory FD, for example, will be comprehended between angles near the 
horizontal $\left(\alpha \simeq 2^{\circ}\right)$ and $\alpha \simeq 30^{\circ}$, and a maximum radius $r$ of approximately $30 \mathrm{~km}$. The approximate maximal height from where the DB can be triggered by the FD is $h$ and $\omega$ is its projection along the DB propagation axis. The zenith angle is represented by $\theta$.

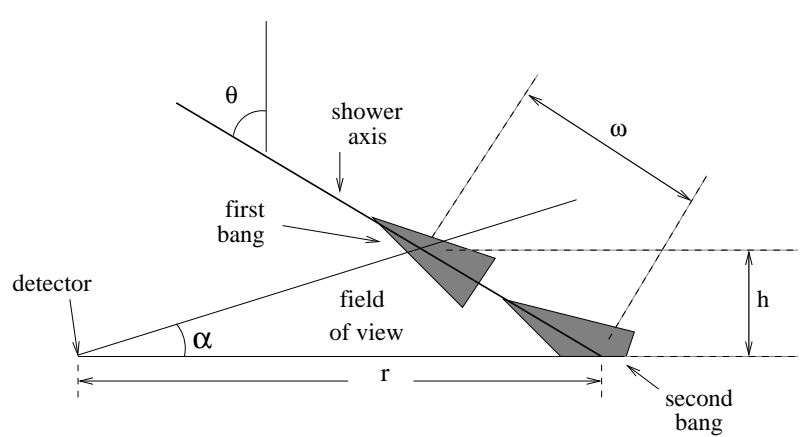

FIG. 1: A schematic view of a Double-Bang and the f.o.v. of the Fluorescence Detector. See text in Section II for details.

The total amount of light emitted by the first EAS is related to the energy transfered to the quark at the moment of the first $v_{\tau}$ interaction, which we define as $E_{1}$. The neutrino energy $E_{\mathrm{v}}$ is divided into the tau energy $E_{\tau}$ and $E_{1}$, i. e., $E_{\mathrm{v}}=E_{1}+E_{\tau}$. For charged current interactions above $0.1 \mathrm{EeV}$, approximately $20 \%$ of the neutrino energy is transfered to the quark [14] and in our calculations we considered it constant. The second EAS, resulting from the tau decay, carries an energy $E_{2}$ of approximately $2 / 3 E_{\tau}$ and may be specially visible when the tau decay is hadronic, which happens with a branching ratio of around $63 \%$ [15]. Therefore, very roughly, we have $\left\langle E_{1}\right\rangle \sim 1 / 5 E_{v}$ and $\left\langle E_{2}\right\rangle \sim 2 / 3\left\langle E_{\tau}\right\rangle \approx 8 / 15 E_{v}$ and the relation between $E_{1}$ and $E_{2}$ is given by: $E_{2} / E_{1} \sim \frac{8}{15} E_{\mathrm{v}} / \frac{1}{5} E_{\mathrm{v}} \approx$ 2.67. The distance traveled by the tau before decaying in laboratory frame is $L=\gamma c t_{\tau}$, where $\gamma=E_{\tau} / m_{\tau}$ and $t_{\tau}$ is the tau mean lifetime, which is presented in Ref. [15] with an error of approximately $0.4 \%$. Therefore, $L \simeq \frac{E_{\tau}}{[\mathrm{EeV}]} \times 49 \mathrm{~km}$ $\simeq \frac{E_{\mathrm{v}}}{[\mathrm{EeV}]} \times 39.2 \mathrm{~km}$.

Now we compare the tau decay length with its attenuation length in the Earth's atmosphere. The energy loss had been calculated [16] including bremsstrahlung, $e^{+} e^{-}$par production and deep inelastic scattering based on a model of the form $-d E / d x=a+b(E) E$ where $a$ is the ionization energy loss and $b$ is the sum of the other contributions due to radiative processes. The second term is dominant above a few $100 \mathrm{GeV}$. So we obtain an attenuation length for the tau in the atmosphere $L_{a}=\left(\rho \sum_{i} b_{i}\right)^{-1} \simeq 33600 \mathrm{~km}$ where $b_{b}=$ $0.08 \times 10^{-7}, b_{p p}=1.4 \times 10^{-7}$ and $b_{d i s}=1.0 \times 10^{-7} \mathrm{~g}^{-1} \mathrm{~cm}^{2}$ from bremsstrahlung, pair production and deep inelastic scattering contributions respectively, and $\rho=1.2 \times 10^{-3} \mathrm{~g} \mathrm{~cm}^{-3}$. Therefore, the attenuation length $\left(L_{a}\right)$ is much longer than the decay length $(L)$ and we do not consider energy loss for the tau propagation.

\section{EVENT RATE}

To calculate the possible number of events in a FD, we consider, for simplicity, one Pierre Auger-like FD with a f.o.v. of $360^{\circ}$. We can write the equation for the $\mathrm{DB}$ event rate:

$$
\frac{d N_{\text {events }}}{d t}=\int_{E_{t h}}^{\infty} d E_{v} \Phi_{v}\left(E_{v}\right) \mathcal{A}\left(E_{v}\right)
$$

where $E_{t h}$ is the minimum detectable energy according to the efficiency of the FD, $E_{v}$ is the $v_{\tau}$ energy, $\Phi_{v}$ is the flux of UHE $v_{\tau}$ at the Earth (which depends on the model of the extra galactic source of high-energy cosmic rays considering maximal mixing), and

$$
\mathcal{A}\left(E_{\mathrm{v}}\right)=\int_{\Omega, A} d \Omega d A P_{\text {int }}\left(E_{\mathrm{v}}, \theta\right) F_{\text {trig }}\left(E_{\tau}, r, \theta\right) \Sigma\left(E_{1}, r\right)
$$

is the acceptance. $\Omega$ and $A$ are the solid angle covered by the detector and the area under the f.o.v. of the detector respectively. $P_{\text {int }}\left(E_{v}, \theta\right)$ is the probability of the $v_{\tau}$ to interact in a given point of the atmosphere, $F_{\text {trig }}\left(E_{\tau}, r, \theta\right)$ indicates the probability of triggering a DB signal and $\Sigma\left(E_{1}, r\right)$ is the efficiency of the FD. We considered only showers moving away from the detector since, otherwise, a large amount of Cerenkov light comes with the fluorescence light, spoiling the data analysis [17].

The interaction probability is given approximately by:

$$
P_{\text {int }}\left(E_{\mathrm{v}}, \theta\right)=\sigma_{C C}^{v N}\left(E_{\mathrm{v}}\right) N_{T}(\chi)
$$

where $\sigma_{C C}^{v N}\left(E_{\mathrm{v}}\right)$ is the average charged current cross section of the neutrino-nucleon interaction and $N_{T}(\chi)$ is the average total number of nucleons per squared centimeter at the interaction point in the atmosphere. $N_{T}(\chi)=2 N_{A} \chi(\theta)$, where $N_{A}$ is the Avogadro's number and $\chi(\theta)$ is the slant depth of the atmosphere within the points where the neutrino must interact to generate a DB event inside the f.o.v. of the FD.

Considering the Earth's curvature, the atmospheric slant depth can be approximately written as:

$$
\chi(\theta, l)=\int_{\lambda} \rho\left(H=l \cos \theta+\frac{(l \sin \theta)^{2}}{2 R}\right) d \lambda,
$$

where $\lambda$ is the path along the arrival direction from the source until the interaction point in the atmosphere, $\rho$ is the atmospheric density, $H$ the vertical height, $l$ is the distance between the interaction point and the ground, along the axis of the EAS (the slant height), and $\theta$, the zenith angle.

Taking the tau decay length $L\left(E_{\tau}\right) \simeq 40 \mathrm{~km}$, for $E_{\mathrm{v}}=1 \mathrm{EeV}$, plus a distance of $10 \mathrm{~km}$ for the second EAS to reach its maximum in the case of a horizontal EAS [16], we estimate the first interaction occur approximately $50 \mathrm{~km}$ away from the detector. In this case it may be difficult to detect the maximum of the first EAS because it will probably develop before reaching the f.o.v. of the FD. We also estimate that if the first interaction happens about $30 \mathrm{~km}$ away from the detector, the maximum of the first EAS can be seen, but on the other hand it may be difficult to detect the maximum of the second EAS which 
probably will reach the ground before its maximal development. To calculate the number of events presented in Section IV we consider the first interaction occurring in a point within $50 \mathrm{~km}$ and $30 \mathrm{~km}$ faraway from the detector.

As the cross section of UHE neutrinos is unknown, usually one adopts the extrapolation of parton distribution functions and Standard Model (SM) parameters far beyond the reach of experimental data. In this way, one can estimate a value for the cross section of the neutrino-nucleon interaction of about $10^{-32} \mathrm{~cm}^{2}$, for energies around $1 \mathrm{EeV}$. Some authors say that this extrapolation gives a too high neutrino-nucleon cross section [18] but others use models that increase this same cross section to typical hadronic cross section values [19]. In this work we use the following cross section parametrization:

$$
\sigma_{C C}^{\nu N}=(5.53+5.52) \times 10^{-36}\left(\frac{E_{\mathrm{v}}}{[\mathrm{GeV}]}\right)^{0.363} \mathrm{~cm}^{2},
$$

which is the SM extrapolation for the neutrinos plus antineutrinos and nuclei cross section in charged current interactions, which have $10 \%$ accuracy within the energy range $10^{-2}<E(\mathrm{EeV})<10^{3}$ when compared with the results of the CTEQ4-DIS parton distributions [20].

We define the trigger factor as:

$$
F_{\text {trig }}\left(E_{\tau}, r, \theta\right)=P_{\text {had }} P_{L} \frac{\omega(r, \theta)}{L\left(E_{\tau}\right)}
$$

where $P_{\text {had }}$ is the hadronic branching ratio of tau decay $\left(P_{\text {had }} \simeq 0.63[15]\right), P_{L}$ is the mean percentage of taus that decay within their decay length in laboratory frame $L\left(E_{\tau}\right)$ and $\omega(r, \theta)$, as can be seen in Fig. 1, is the approximate size of the shower axis inside the f.o.v. of the detector where the vertical plane containing the shower axis passes through the center of the FD. In Eq. 6, we are imposing $\omega(r, \theta) / L\left(E_{\tau}\right)=1$ if $\omega(r, \theta)>L\left(E_{\tau}\right)$ so that we have a conservative estimation of the trigger factor. Since we are using a fixed value of the decay length $L\left(E_{\tau}\right)$ for a given energy, corresponding to the length traveled by the tau in one lifetime, we find $P_{L}=1-e^{-1} \simeq 0.63$, because it is expected that a fraction $1 / e$ of the taus do not decay within the length $L$.

The efficiency of the FD was estimated as:

$$
\Sigma\left(E_{1}, r\right)=\Upsilon \Sigma^{\prime}\left(E_{1}\right) \Sigma^{\prime \prime}(r)
$$

where $\Upsilon$ is the fraction of the time the fluorescence detector operates $(\Upsilon \simeq 0.1$ because the fluorescence detector can only operate in clear moonless nights), $\Sigma^{\prime}\left(E_{1}\right)$ is the efficiency depending on the energy of the first EAS of the DB Phenomenon, that is less energetic than the second one, and $\Sigma^{\prime \prime}(r)$ is the efficiency depending on the distance from the FD to the EAS core where it reaches the ground. $\Sigma^{\prime}\left(E_{1}\right)$ and $\Sigma^{\prime \prime}(r)$ depend on the characteristics of each detector. For $\Sigma^{\prime \prime}(r)$ we used a Gaussian distribution centered at $r=12.5 \mathrm{~km}$ faraway from the detector and variance of $5.0 \mathrm{~km}$. We analyze two $\Sigma^{\prime}\left(E_{1}\right)$ cases. For the first one we consider $\Sigma^{\prime}\left(E_{1}\right)$ rising logarithmically from 0 to 1 in the energy range between approximately $0.3 \mathrm{EeV}<E_{1}<30 \mathrm{EeV}$. This situation is consistent with the characteristics of a Pierre Auger-like FD. In the second case we consider $90 \%$ efficiency $\left(\Sigma^{\prime}\left(E_{1}\right)=0.9\right)$ for neutrino energies between 0.5 and $5 \mathrm{EeV}$. The behavior of $\Sigma^{\prime}\left(E_{1}\right)$ can be seen in Fig. 2 in terms of $E_{v}$ for both cases presented above.

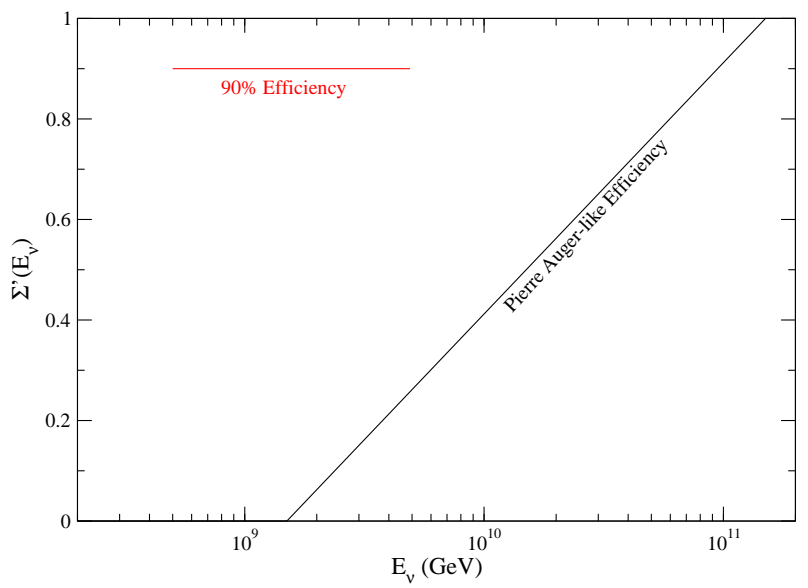

FIG. 2: Efficiency as a function of the neutrino energy. The Pierre Auger-like efficiency was used to calculate the event rate shown in columns $N_{1}, N_{2}$ and $N_{3}$ of Table I, and the $90 \%$ efficiency was used to calculate the number of events for two FD's with $60^{\circ}$ f.o.v. shown in the last column of Table I.

\section{RESULTS}

Using Eq. 1 with all the phenomenological considerations given above, we calculate the expected DB event rate which can be seen in Table I for different models and limits of UHE cosmic ray flux, and in different energy intervals. The last column of Table I shows the event rate in a hypothetical case with $90 \%$ efficiency in the more relevant energy range for DB events $\left(0.5 \mathrm{EeV}<E_{\mathrm{v}}<5 \mathrm{EeV}\right)$, using 2 FD's with $\alpha=60^{\circ}$ (see Fig. 1).

From Table I one can learn which is the energy interval which is relevant to detect DB events with a Pierre Augerlike FD. The models WB [24] and MPR [25] are limits for the UHE neutrino flux based on cosmic ray observation. Both consider the neutrinos coming from the interactions of protons and photons in the sources generating pions that will decay into muons, electrons and neutrinos. The basic difference is that the authors of the WB limit state that the sources are completely transparent to the protons and on the other hand the authors of MPR limit say that the sources might have some opacity to the protons that generate neutrinos in the interactions with the ambient light in the source. So there could be some neutrino flux that arrive at the earth but it might not be associated with the cosmic ray flux observation. A reasonable flux model might predict an event rate between these two limits.

Figure 3 shows the dependence of the acceptance with the zenith angle. It is given by the integrand of Eq. 2 integrated in $d A$ and for arbitrary neutrino energy. The acceptance is higher for events coming from almost horizontal angles, but it is significant even for angles around 60 degrees. 
TABLE I: Number of events in the Pierre Auger-like FD during a period of one year, calculated in different regions of the energy spectrum and for different models and limits of cosmic ray flux. The last column was calculated with different considerations on the FD characteristics. See text for details. TD-92 stands for the model in reference [21]; TD-96 for the model in reference [22]; AGN-95J for the model in [23]; WB for [24] and MPR for [25].

\begin{tabular}{llll|l}
\hline \hline Models & $N_{1}{ }^{a}$ & $N_{2}{ }^{b}$ & $N_{3}{ }^{c}$ & $N_{4}{ }^{d}$ \\
\hline TD-92(0) & 1.83 & 0.46 & 0.03 & 118 \\
TD-92(0.5) & 0.03 & 0.01 & 0.001 & 1.46 \\
MPR & 0.005 & $9.0 \times 10^{-4}$ & $3.7 \times 10^{-5}$ & 0.48 \\
TD-92(1.0) & 0.004 & 0.002 & $3.2 \times 10^{-4}$ & 0.093 \\
TD-92(1.5) & 0.002 & $7.1 \times 10^{-4}$ & $1.3 \times 10^{-4}$ & 0.037 \\
AGN-95J & $6.1 \times 10^{-4}$ & $1.1 \times 10^{-4}$ & $4.7 \times 10^{-6}$ & 0.060 \\
WB & $1.1 \times 10^{-4}$ & $2.0 \times 10^{-5}$ & $8.4 \times 10^{-7}$ & 0.011 \\
TD-96 & $4.7 \times 10^{-8}$ & $4.8 \times 10^{-9}$ & $8.3 \times 10^{-11}$ & $9.0 \times 10^{-6}$ \\
\hline
\end{tabular}

${ }^{a} E_{\mathrm{v}}>1.0 \mathrm{EeV}$

${ }^{b} E_{V}>10 \mathrm{EeV}$

${ }^{c} E_{\mathrm{V}}>100 \mathrm{EeV}$

${ }^{d}$ Two FD's with $\alpha=60^{\circ}$ and $90 \%$ efficiency for energies $0.5 \mathrm{EeV}<E_{\mathrm{v}}<5$ EeV.

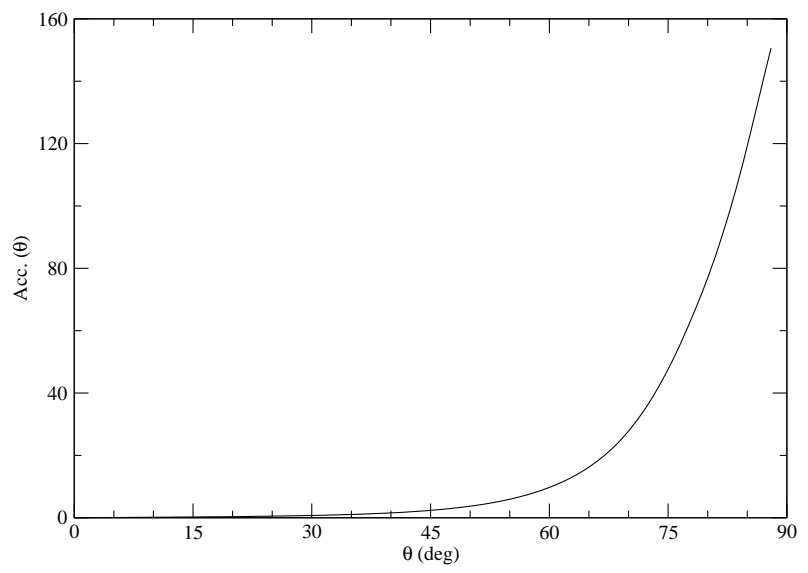

FIG. 3: Dependence of the acceptance with the zenith angle. The energy and vertical axis scale are arbitrary.

\section{BACKGROUND EVENTS}

We consider here the possibility of a particle of the cosmic radiation to masquerade a DB event depending on the accuracy of the detector. The probability of a proton, for example, to generate two EAS's and masquerade the DB generated by a neutrino depends mainly on two possibilities: 1) the primary proton interaction generates some fragment that will give rise to a secondary shower deep in the atmosphere with energy higher than the first one. 2) another shower created by some independent particle interacts deep in the atmosphere masquerading the second EAS of the DB.

In the possibility 1 , the second EAS will be created by the decay or interaction of the fragment deep in the atmosphere. Usually the primary proton generating an EAS loses roughly half of its energy to the secondary particles that constitute the
EAS and therefore it is unlikely that a second EAS has more energy than the first one. There may be some cases where the proton loses only a few amount of its initial energy to the EAS and that a high-energy fragment created by the proton decays or interacts creating a second EAS with energy higher than the first one. It could generate a possible background for DB events with angles between $60^{\circ}$ and $70^{\circ}$, so it has to be carefully studied. Now, taking into account that for energies of the order of $1 \mathrm{EeV}$ we have a cosmic ray flux of less than 1 particle per $\mathrm{km}^{2}$ per year and that the only particles that could probably interact deep in the atmosphere are neutrinos, generating the second independent EAS near the detector, the chance that the primary particle and this second independent neutrino come from the same solid angle direction, in a time interval of the tau mean lifetime in the laboratory frame of $\gamma t_{\tau} \approx 131 \times \frac{E_{\tau}}{[\mathrm{EeV}]} \mu \mathrm{s}$ is approximately 1 in $10^{12}$, which excludes the possibility 2 . The direction of the two EAS's can be identified specially if two FD's trigger the same DB event (with only one detector, it is difficult to know the direction of the EAS in the plane that contains the EAS and the detector).

Based on these assumptions, $E_{2} / E_{1}$ may be a good parameter to identify DB events if the measured energies from the two EAS's are accurate enough. The error in the energy measured by a FD depends mainly on the atmospheric conditions but hardly will exceed $50 \%$. One can be optimistic when trying to identify a DB because the relation between the energies of the two EAS's is more important than the absolute energy of each EAS. We can make a conservative estimation of the error in the average ratio $E_{2} / E_{1}$ considering the error in the absolute energy of $50 \%$. This will give a relative error to the energy ratio of $70 \%$. Even considering such an error the energy ratio $E_{2} / E_{1}>1$ cover all the events with $E_{2} / E_{1} \approx 2.67$, the energy relation expected for DB events as deduced in Section II. Then $E_{2} / E_{1}>1$ could be one minimal condition to identify DB events.

Finally, we have to mention that low energy $v_{\tau}$ 's will generate taus which decay before the complete development of what would be the first EAS. In such a situation the so called DB event looks like a single EAS generated by a proton. We estimate that events with $E_{E A S} \sim 0.1 \mathrm{EeV}$ can fall into this possibility and have to be studied carefully.

\section{CONCLUSION}

Taking into consideration neutrino oscillations, one expects that one third of the high-energy neutrino flux arriving at the Earth should be composed of $v_{\tau}$ 's. These neutrinos can interact in the Earth's atmosphere generating a DB event. Many recent papers [16, 26-30] analyze the potential of the Pierre Auger Observatory and other experiments to detect almost horizontal air showers generated by UHE neutrinos. We investigate the potential of a Pierre Auger-like FD to observe DB events.

In Table I we presented numbers of DB event rate per year expected for a Pierre Auger-like FD and also for an optimistic hypothetical case with 2 FD's, $\alpha=60^{\circ}$ (see Fig. 1) and $90 \%$ efficiency for neutrino energies between 0.5 and $5 \mathrm{EeV}$. 
For the Pierre Auger-like efficiency case, only the topological defect model TD-92(0) predicts a significant number of DB events, of 1.83 per year for neutrino energy bigger than $1 \mathrm{EeV}$. On the other hand, assuming the very feasible configuration with 2 FD's and 90\% efficiency for neutrino energies between 0.5 and $5 \mathrm{EeV}$, models like TD-92(0), TD-92(0.5) and also MPR limit can be tested predicting, respectively, 118, 1.46 and 0.48 events per year.

DB events have very particular characteristics. Different from the neutrino events in surface detectors, DB events do not need to come from the very near-horizontal angles. Despite the low probability of interacting at the top of the atmosphere, we can also have $v_{\tau}$ 's creating DB events with incident angle of approximately $60^{\circ}$ or larger. In the Pierre Auger Observatory DB events may also have a lower primary neutrino energy, around $1 \mathrm{EeV}$, different from the energies around $50 \mathrm{EeV}$ and beyond expected for an ordinary EAS generated by the highest energy cosmic rays.

The energy range where the DB can be detected is very strict. For EAS energies less then $0.3 \mathrm{EeV}$ the efficiency of the Pierre Auger Observatory FD may be too low and for $\nu_{\tau}$ energies greater then $20 \mathrm{EeV}$ the two EAS's are too separated. In the $v_{\tau}$ energy range between approximately $2 \mathrm{EeV}$ and $10 \mathrm{EeV}$ a considerable part of the two EAS's that characterize a DB may be detected and then we could have a DB trigger on the Auger Observatory. An alternative method to study some aspects as possible background events and the expected shape of this kind of phenomenon is using Monte Carlo simulations with tau and $v_{\tau}$. This would account, for example, for fluctuations in the EAS maximum that would make the observation of DB events even more difficult.

Despite of the fact the DB Phenomenon may be very rare, it is very important to be prepared for its possible detection, specially in case the Pierre Auger ground array detects nearhorizontal air showers which points to the presence of electron and/or muon neutrinos. Consequently oscillations imply a considerable number of $v_{\tau}$ too. With such a motivation, the Auger Observatory trigger could be calibrated to be more sensitive for energies around $1 \mathrm{EeV}$. With good efficiency in this energy range, more detectors and more years collecting data, we could have more significant statistics.

\section{Acknowledgments}

Conselho Nacional de Desenvolvimento Científico e Tecnológico - CNPq, scholarship 140241/2001-9.
[1] K. Greisen, Phys. Rev. Lett. 16, 748 (1966).

[2] G. T. Zatsepin and V. A. Kuz'min, JETP Letters 4, 78 (1966).

[3] F. Halzen and D. Saltzberg, Phys. Rev. Lett. 81, 4305 (1998).

[4] F. Halzen, Lectures on neutrino astronomy: Theory and experiment, Lectures presented at the TASI School (1998), astro$\mathrm{ph} / 9810368$.

[5] J. G. Learned and S. Pakvasa, Astropart. Phys. 3, 267 (1995).

[6] P. C. Holanda and A. Y. Smirnov, Phys. Rev. D 66, 113005 (2002).

[7] G. L. Fogli, E. Lisi, and A. Marrone, Phys.Rev. D 64, 093005 (2001), hep-ph/0105139.

[8] K. Eguchi et al., Phys. Rev. Lett. 90, 021802 (2003).

[9] S. H. Ahn et al., Phys. Lett. B 511, 178 (2001).

[10] S. H. Ahn et al., Phys. Rev. Lett. 90, 041801 (2003).

[11] H. Athar, Chin. J. Phys. 42, 1 (2004), hep/ph-0308188.

[12] S. Eidelman et al., Phys. Lett. B 592, 251 (2004), pdg.

[13] Pierre auger project design report (1997), http://www.auger.org/admin/DesignReport/index.html.

[14] C. Quigg, M. H. Reno, and T. P. Walker, Phys. Rev. Lett. 57, 774 (1986)

[15] S. Eidelman et al., Phys. Lett. B 592, 414 (2004), pdg.

[16] X. Bertou et al., Astropart. Phys. 17, 183 (2002), astro$\mathrm{ph} / 0104452$.

[17] J. A. Bellido, Tech. Rep. GAP-98-027, Auger Colaboration (1998), http://www.auger.org/admin-cgibin/woda/gap_notes.pl/.
[18] A. Kusenko and T. Weiler, Phys.Rev.Lett. 88, 161101 (2002), hep-ph/0106071 v2.

[19] A. Jain et al., Int. J. Mod. Phys. A17, 533 (2002), hep$\mathrm{ph} / 0011310 \mathrm{v} 2$.

[20] R. Gandhi, C. Quigg, M. H. Reno, and I. Sarcevic, Phys. Rev. D 58, 093009 (1998).

[21] P. Bhattacharjee, C. T. Hill, and D. N. Schramm, Phys. Rev. Lett. 69, 567 (1992).

[22] G. Sigl, Space Science Reviews. 75, 375 (1996).

[23] K. Mannheim, Astropart. Phys. 3, 295 (1995).

[24] J. Bahcall and E. Waxman, Phys. Rev. D 64, 023002 (2001), hep-ph/9902383 v2.

[25] J. P. Rachen, R. J. Protheroe, and K. Mannheim, Phys. Rev. D 63, 023003 (2001), astro-ph/9812398 v3.

[26] K. S. Capelle et al., Astropart. Phys. 3, 321 (1998), astro$\mathrm{ph} / 9801313$.

[27] H. Wilczyński, Tech. Rep. GAP-00-020, Auger Colaboration (2000), http://www.auger.org/admin-cgibin/woda/gap_notes.pl/.

[28] M. Ave et al., Astropart. Phys. 14, 109 (2000), astro$\mathrm{ph} / 0003011$.

[29] J. L. Feng et al., Phys. Rev. Lett. 88, 161102 (2002), hep$\mathrm{ph} / 0105067$.

[30] C. Aramo et al., Astropart. Phys. 23, 65 (2005), astro$\mathrm{ph} / 0407638$. 